\title{
Program evaluation in a cross-cultural context: Action research, program logic and youth justice in Thailand
}

\begin{tabular}{c|c}
\hline Matthew J Willis & Adam M Tomison \\
Australian Institute of Criminology & Australian Institute of Criminology \\
Honorary University Fellow, Northern \\
Institute, Charles Darwin University \\
matthew.willis@aic.gov.au
\end{tabular}

Keywords: cross cultural program logic, participatory action research, cross cultural evaluation, multilingual evaluation, juvenile justice evaluation, Juvenile Justice Reform Project, Justice for Our Youth project.

\begin{abstract}
Using the evaluation of a multi-faceted juvenile justice project as a case study, we demonstrate how applying an action research approach to program logic development provided a way of arriving at shared understandings of evaluation in a cross-cultural, cross-language context. The paper explores work undertaken by the Australian Institute of Criminology for the Thailand Department of Juvenile Justice and Observation to support the evaluation of the Justice for Our Youth (JOY) project, a complex project aimed at improving outcomes for young offenders by improving the quality of service offered by the Department. We describe a workshop conducted in Thailand where the authors provided capacity-building for Thai officials in program monitoring and evaluation and then worked with the officials to apply the learning from this part of the workshop to developing program logic models and identifying data and information needs for the JOY program evaluation. The utility of Participatory Action Research (PAR) and program logic approaches to working in a cross-cultural, cross-language context are discussed and their application to other cross-cultural situations is considered. The authors conclude that PAR can provide a valuable and appropriate model for establishing mutual understanding and trust in such contexts, but also recognise that the realities of difference and distance can reduce the ability of evaluators to apply PAR in a way that represents best practice.
\end{abstract}




\section{Introduction}

There are many publications and manuals that attempt to provide ideal or best practice approaches to conducting evaluations in community and cross-cultural settings. The reality of evaluation practice is that often the 'ideal' is not possible or practicable, with evaluators constrained in terms of project parameters, resources (time, funding and access to participants) and the approaches able to be employed. Typically, evaluators must 'cut the cloth' of the evaluation to take into account imposed constraints and other conditions present in the evaluation environment, while maintaining an ethical approach, doing their best to meet the needs of stakeholders and participants and enabling sufficient data to be collected to demonstrate project success.

In this paper the authors demonstrate the application of an action research approach based around the development and use of program logic models, as part of an evaluation of a multi-faceted juvenile justice project being developed in Thailand. By describing the process used, the paper aims to provide insights about the benefits and limitations of Participatory Action Research approaches to generating shared understandings of evaluation in a cross-cultural, multi-language context. The aim is to give a real world (in situ) example of conducting evaluation in such contexts to inform evaluation practitioners and others involved with cross-cultural engagement. Some insights are provided into how the practice of evaluation can occur successfully in a real world situation where situational constraints affect both the evaluators and participants.

\section{Background}

The focus of this paper is an evaluation project being conducted by the Australian Institute of Criminology (AIC) for the Thailand Department of Juvenile Observation and Protection (DJOP). The DJOP provides services for children and young people entering the justice system, including juvenile detention and post-release services. The Department is empowered under Thai legislation to undertake investigation of juveniles' circumstances and characteristics and assessment to determine individual service and support needs.

Between 2009 and 2013 DJOP, together with a number of other Thai agencies, implemented the Juvenile Justice Reform Project (JJRP), which aimed to address service improvements in screening and classification, rehabilitation programs, pre-release preparation and post-release support. This Project resulted in the development of a range of assessment and classification tools, intervention programs for juveniles at each stage of the juvenile justice process and a network of government and non-government agencies providing services and programs for young offenders.

Consultation and analysis following the Juvenile Justice Reform Project identified areas where further work was needed to realise the outcomes established through the Project. A needs analysis showed that a majority of Probation Officers lacked the knowledge and skills to properly use the risks and needs assessment structured interview form developed through the JJRP and some were reluctant to use the form. The analysis also showed DJOP lacked standardised objective psychological assessment tools to 
assess mental health problems and traits associated with the criminal behaviours of young people. The Department also lacked a fully developed throughcare - focused intervention program provided for juveniles in detention training schools. 'Throughcare' refers to the treatment and supports provided to prisoners and detainees from their reception into custody that continue after release into the community (Borzycki \& Baldry, 2003). Finally, the intervention programs being used did not fully address criminogenic needs and risks for the young people, and did not utilise community resources, leading to extended periods in residential placements and have inadequate continuing care after release.

The Justice for Our Youth (JOY) project was created to fill gaps remaining after JJRP. JOY was established explicitly around principles of enhancing the participation of stakeholders and greater involvement of DJOP staff, which issues were identified through the JJRP process. There are three sub-projects within the overall JOY project:

1. Building staff capacity in risk and needs assessment through a program of training for experienced Probation Officers, and the solidification and transfer of skills from these Officers to less experienced Probation Officers.

2. Development of mental health and behavioural assessment tools for juveniles with mental health issues and complex problems (through standardized adaptation of existing standard psychometric tests developed outside Thailand).

3. Development of seamless intervention and rehabilitation programs for juveniles (using an established approach known as to provide throughcare outcomes.

The JOY project commenced in mid-September 2013 and will continue for approximately three years, until mid-October 2016.

\section{The Evaluation Process}

Achieving quality evaluation outcomes requires the use of evaluation processes that are well-grounded and well-designed. Effective practice principles suggest that each evaluation should be tailored to fulfil the specific purpose for which it is required and to meet the needs of the various stakeholders involved (Stufflebeam, 2004, Tomison, 2000). Often there are multiple purposes behind the evaluation, multiple dimensions to the intervention or program being evaluated, and multiple stakeholders whose needs must be considered. Different stakeholders will often have disparate interests and may well require different evaluation 'products' and styles of communication (Williams \& Tomison, 2013).

The research question(s) and the level of explanation required determinethe methodologies and research tools used and the degree of experimental rigour that is desired and/or possible (Frechtling, 2007; Funnell \& Rogers, 2011; Tomison, 2000). The type of data and information required to answer the research questions, and the practicalities of gathering the necessary data and information, are also essential in shaping the methodology to be 
applied to each evaluation. Not infrequently, interventions may be hampered by limited access to statistical data or records. Sometimes, what is available may not capture the reality of participants' experiences or the issue under consideration. Nevertheless, funding bodies and other external stakeholders often require quantitative measures, "hard data', to ensure the methodological rigour of any findings, and to enable comparisons over time and between programs and/or communities. The challenge when undertaking work in applied community settings, particularly when working with local communities of different cultural and language backgrounds, is creating evaluation evidence that is meaningful to funding bodies and other stakeholders. The use of multiple methods and data sources to 'build a picture' over time typically improves the ability of the evaluation to demonstrate program success (Pawson \& Tilley, 1997; Tomison, 2004).

In order to establish the key questions, measures and sources of data and information for programs and evaluations, many evaluators use a variant of a 'program logic' or 'theory of change' framework (e.g. Clark \& Anderson, 2004; Funnell \& Rogers, 2011). Program logic is a process of developing a conceptual model of how, and why, a program is expected to work (Funnell \& Rogers, 2011; McLaughlin \& Jordan, 1999). While models can vary, program logic modelling typically results in a diagram that depicts the inputs and activities required for the program to operate, the assumptions and external factors that influence the program's development and implementation, and the outcomes expected from the program in the short-term, medium-term and long-term (McLaughlin \& Jordan, 1999). Program logic models can be valuable tools for communicating and disseminating information about a program, identifying the key elements of a program, informing measure of success, and for identifying data and information needs (McLaughlin \& Jordan, 2004).

Program logic frameworks are necessarily influenced by those who are involved in their development. Frameworks developed in isolation by the evaluator and the staff of an intervention or program may lack the depth, rigour and application to specific contexts and circumstances that can be achieved if a wider group of stakeholders, including community participants, are involved in the framework development process. This is particularly important where there are cultural or language differences between the evaluator/researcher, community members and other stakeholders.

Ideally then, a shared understanding of key concepts and intentions should be developed between the evaluator(s) and the range of stakeholders for the evaluation - this will be critical to the evaluation's success. Taking a culturally sensitive approach to building this shared understanding will also help to overcome some of the potentially negative consequences of conducting cross-cultural evaluation and research. These can include imposing Western perspectives and conceptual frameworks on the evaluation process and its findings, reducing the non-Western participants to being passive objects of examination, and developing findings based on shallow information and flawed or limited understandings (see Liamputtong, 2010).

A key element for the AIC in conducting an effective evaluation of JOY was to ensure that a culturally respectful process was developed that was flexible enough to be used with the range of diverse professional groups involved with the project (and the 
evaluation), which considered their varied experiences as participants in evaluations. The process respected the expertise of the DJOP staff and their knowledge of the context and circumstances in which the JOY project was developed and implement. Key considerations in framing the project including ensuring the aims, outcomes and success measures of the project were accurately defined and that all participants:

- shared an understanding of the project, its aims and objectives;

- agreed (through a collaborative process) on the nature and goals of the evaluation;

- contributed to developing an agreed understanding about how and why data would be collected; and

- had roles in the evaluation process that aligned with their knowledge and experience and allowed them to contribute to the overall success of the JOY project.

\section{Frameworks}

The evaluators began the initial steps of building a framework for the evaluation using mixed methods approaches within a realist evaluation framework (Pawson \& Tilley, 1997). A combination of quantitative and qualitative methodologies appeared to present the best option for capturing the data and information needed for the evaluation. Quantitative methodologies would support the need for highly tangible datadriven measures, such as reductions in offending behaviour that are typically sought by evaluation stakeholders. Qualitative methodologies would allow for the capture of deeper levels of information about outcomes, such as changes in perceptions or the quality of engagement between DJOP and young people. The evaluators considered that qualitative techniques would be particularly valuable given the crosscultural context of the evaluation. Qualitative approaches support the gathering of information linked to subjective experiences, situational meanings and allow for levels of interpretation and flexibility that can help to bridge gaps between parties in crosscultural situations (Liamputtong, 2010).

In circumstances where researchers and evaluators come from positions of cultural dominance, traditional positivist methodologies can override the perspectives of participants and deny them agency (Liamputtong, 2010). This typically occurs when the evaluand is a program or intervention involving Indigenous (first nations) peoples, who come to the research or evaluation process from an inherently disempowered position. The AIC evaluators considered that qualitative methods, used within a realist evaluation framework, would support the agency of the Thai staff participating in the evaluation. At the same time realist approaches, with their emphasis on understanding context and refining theories of how an intervention produces change, offered the best prospect for collaboratively generating knowledge of the project and the environment within which it is implemented (Pawson \& Tilley, 2007). 


\section{Participatory Action Research}

The evaluators' initial considerations for the evaluation framework were based primarily on reading of documents describing the basis and establishment of the JOY project. The AIC and DJOP agreed that further development of the framework and implementation of the evaluation process should be realised through collaboration between the parties. While mixed methods approach and realist perspectives appeared to the evaluators to provide an appropriate basis for the evaluation, progressing without forming a strong collaborative approach would undermine the inclusionary objectives that these methods and perspectives were intended to support.

A Participatory Action Research (PAR) approach was chosen as the foundation methodology for working with program participants to develop an agreed program logic model that would inform and support evaluation of the JOY project and its subprojects. PAR is an approach to research that brings together those directly involved in an intervention or project that is being evaluated - generally staff of an organisation or members of a community - and those tasked with conducting the evaluation (Whyte, Greenwood \& Lazes, 1991). Using PAR approaches, direct stakeholders are involved throughout the evaluation, from initial planning and design to interpretation and dissemination of the findings. Based on a fluid process founded on iterative cycles of Planning, Acting, Observing and Reflecting; PAR has been found to be a very useful approach for such human service evaluations, particularly where the focus is on program and service improvement rather than accountability. PAR supports the active engagement of those directly involved with delivering services and allows their knowledge and experience to contribute directly to the foundations of the evaluation (Brydon-Miller, Kral, Maguire, Noffke \& Sabhlok, 2011; Burns, 2006; Crane \& O’Regan, 2010; Hecker, 2008; McTaggart, 1989; Stringer, 2014).

Such approaches allow project teams to develop their own evaluation frameworks and ways of working. Having enacted planned actions, PAR explicitly encourages teams and their members to build in processes for collecting their observations and experiences of practice, to reflect on them with their team (i.e., to assess what is working or not working, or how can the project or outcomes be improved) and using the outcomes of these processes to guide a continuous improvement approach to program delivery and evaluation (Kemmis \& McTaggart, 2000; McTaggart, 1989).

PAR is based on the development of a partnership between the evaluator, funding body and participants, with participants taking an active role in developing and informing the evaluation. That is, the intent is that project teams 'own' their projects and the evaluation. PAR is expressly designed to be participatory and collaborative, with evaluators in the role of 'walking alongside' and 'doing with' rather than 'doing to' a project. The notion of 'doing with' embraces the fundamental idea that project teams are not merely objects of examination for the evaluation, or passive actors whose outputs are scrutinised, but active participants in the process of evaluating. 
While PAR is most often used with qualitative research, it fits with a multiple methods approach and is flexible enough to underpin many evaluation models and approaches, and the inclusion of quantitative data where it is available, as was the case for the JOY project. The action research approach is also well-suited to operating in a range of cultural contexts, and facilitates culturally secure evaluation practice by its very nature of being designed around inclusionary processes (Brydon-Miller et al. 2011; Hecker, 2008; McTaggart, 1989).

\section{Evaluating the JOY Project, Thailand}

The Australian Institute of Criminology (AIC) conducts criminological research across a range of topic areas, including evaluations of crime-related interventions and programs. During 2013, the Thailand DJOP contacted the AIC to commence a process of engaging the AIC to provide expertise to support the evaluation of the JOY project. Through negotiations it was agreed that the AIC would conduct two workshops in Thailand to build the capacity of DJOP staff in program monitoring and evaluation, to improve their understanding and ability to collect data and information for the evaluation of JOY, to undertake the data analysis for the evaluation; and to prepare an evaluation report of JOY and its three sub-projects.

In February 2014, the authors travelled to Bangkok to deliver a workshop to approximately twenty DJOP staff, representing most of the staff working on the JOY project. The primary aim of the workshop was to build the capacity of DJOP staff in program monitoring and evaluation and to develop program logic models for each of the JOY sub-projects with the active involvement of program participants. The first part of the workshop focused on general principles and best practice issues, while later sessions adopted an action research approach to apply these principles and practices to developing evaluation frameworks for each of the three sub-projects.

The workshop helped to refine both AIC and DJOP understandings about the aims of the JOY sub projects, their intended outcomes, how the success of these outcomes could be measured and what data and information could be collected to inform these measures. The workshop also represented a critical milestone in the application of an action research approach for the development, implementation and evaluation of the JOY project.

Explicit intended outcomes of the process were for staff participants to develop:

- a shared understanding of the role each person would play;

- the importance of that role for achieving both program and evaluation objectives;

- a clear understanding of which information would be collected, how it would be collected and who would be responsible for its collection; and

- how that information would be used to populate the evaluation framework and program logic model. 
This initial 'skilling up' and capacity-building for staff participants formed the basis for establishing a positive engagement with AIC staff allowing for further relationshipbuilding to occur over time. It also enabled the building of relationships between AIC and DJOP staff and assisted in establishing the trust and mutual understanding needed to support the active exchange of information and sharing of insights fundamental to a PAR approach.

Conducting the workshop required a quite measured and structured form of delivery from the AIC facilitators, as most of the DJOP staff had little or no understanding of English. Throughout the three days, the workshop was continually translated into Thai by the head of the DJOP research section, who was also leading the JOY project. The AIC facilitators used strategies such as re-stating and questioning to regularly check for clear understanding of the information being provided. Throughout the workshop there were periods where the DJOP staff discussed issues among themselves in Thai, providing only summaries of the discussions in English. Welcoming these discussions, rather than trying to impose a requirement that all discussions be translated into English was both respectful to the DJOP staff and supportive of richer levels of engagement by the Thai speakers than would otherwise have been possible.

\section{Program logic and PAR}

Following initial training and capacity-building sessions, the workshop was then focused on working with DJOP participants to describe and refine how the evaluation would operate in practice, using PAR and the development of program logic models for each of the three JOY sub-projects. Before each session, the facilitators prepared a program logic diagram that was partly completed on the basis of information known to the facilitators through background documentation on JOY (translated from Thai into English), together with knowledge of long-term criminal justice outcomes drawn from experience on other evaluations.

This proved to be very useful in a number of ways. In a purely practical sense, having the diagrams part-completed helped move the discussion along and overcame the time constraints that came with working across languages. Information that had been prepared in a succinct way suited to the model could be directly interpreted and allowed discussions around the elements of the model to proceed from a clearer foundation. Part completion also allowed those unfamiliar with logic model approaches before the workshop to engage more readily with concept of program logic by presenting those concepts in a more tangible way than a blank template would have allowed. Working from partial models also indicated in an explicit manner the extent of the facilitators' familiarity with the resources, influences and external environmental factors affecting the project and the types of data and information that could be useful for the evaluation. While this approach helped to establish the facilitators' credibility and knowledge of youth justice systems; providing incomplete program logic models that required 
the stakeholder input and evaluator/participant discussion for completion proved to participants how crucial their input and expert knowledge was to developing the evaluation.

Discussions held during the workshop allowed the evaluators to gain insights into the forms of information and data held on young offenders to support case management, internal reporting between DJOP head office and the provinces, and DJOP's relationships with other criminal justice system agencies. The discussions also shed light on DJOP's expectations of its staff and the ways in which DJOP staff engaged with the families of young offenders. Understanding the expectations of staff was important for the evaluation as the anticipated outcomes of two of the sub-projects include improvements in the knowledge and skills of DJOP staff.

Further, in evaluating any staff-delivered intervention, it can be valuable to understand the organisation's expectations of its staff as this will help evaluators establish performance measures and assess whether staff have met organisational targets in implementing the intervention. This may be less of an issue when evaluations are conducted within the same cultural group. In the absence of specific information assumptions will generally be made about organisational expectations based on societal norms. These expectations can then be tested at later stages of the evaluation. When working across cultures, particularly with different systems of government, it becomes more critical for evaluators to understand the contributions of organisational perceptions and societal norms. Being able to gain some insights into how the DJOP saw its relationships with young people and their families, and how the organisation viewed the responsibilities of young offenders, was important for identifying sources of information for the evaluation. The overall objectives of the JOY project include reduced juvenile offending, as well as better life outcomes for young offenders. These outcomes would include reintegration with families and communities and engagement in education or employment. While these outcomes would be familiar to evaluators working with the Australian criminal justice system, discussions allowed the evaluators to identify subtle differences in the Thai context related to differing expectations about when and how young people would engage with education and employment. These differences were able to be captured and will be explored further, using PAR approaches, as the evaluation progresses.

JOY aims to achieve its objectives through improved juvenile justice services, such as individualised case management services delivered from the young person's first contact with the agency through to their return to the community, including support in the community. Young people are clearly integral to the work of DJOP and being able to include their voices, and those of the families and communities they return to, is an important element of ensuring a well-rounded evaluation. It is also important that DJOP is able to provide the evaluators with qualitative and quantitative evidence of outcomes in the form of risk assessments and case management plans that can be analysed to identify the impacts of the JOY sub-projects. The insights gained through the workshop assisted the evaluators to understand how they, and DJOP, could access information from and about young Thai people. These insights have informed questions about how indicators of successful reintegration to communities should be 
conceptualised and measured in the context of Thai family structures and familial and societal expectations of young people and their roles.

Overall, the workshop provided an ideal starting point for applying PAR approaches to this evaluation project. The workshop became a tangible manifestation of the PAR model of reflection, data/information collection and action (see Baum, MacDougall \& Smith, 2006). Participants were able to reflect on aspects of the JOY project, its outcomes and information needs and a number noted how much they had learned about their own project through this reflective process. The process provided a platform for sharing information about the project and the work of the DJOP; some of this information then required action to adjust the program logic models and data collection matrix. For example, information concerning the program's objectives and expected outcomes that had been necessarily included in the project establishment documentation created for the funding provider, were not necessarily able to be operationalised in a way that could be measured. Within the workshop context, the facilitators and DJOP participants were able to brainstorm data collection options that would be achievable (and better placed) ways of meeting the needs of key stakeholders. Using a PAR approach, these options were incorporated into the logic models and led to a new cycle of reflection, collection and action.

The PAR cycles occurring within the workshop served as a microcosm of the PAR processes that will be applied throughout the evaluation, both by DJOP and the AIC. The authors anticipate that a PAR approach will help both organisations manage the substantial challenges of implementing a long-term multi faceted project with wideranging outcomes and diverse stakeholders, and evaluating this project in a crosscultural, cross-language and geographically distant context.

\section{Participation and Power}

One of the distinctive features of PAR is that it allows for open and respectful communication that bridges power relationships by deliberately sharing power between the parties and imparting equal value on the contributions of researchers and those being researched (Baum, MacDougall \& Smith, 2006). This makes the approach highly suitable for working in a cross-cultural situation where the researchers or evaluators are not necessarily fully versed with cultural protocols or expectations. PAR approaches help to make explicit the notion that an external evaluator is bringing a particular knowledge or expertise to the relationship, but is not doing so from a position of superiority.

In the case of working with stakeholders in an Asian country, this helps to overcome any notion that Western perspectives may be inherently superior, or that the Asian partner does not have knowledge or expertise to contribute to the working relationship. In the case of evaluations involving programs operating in Australian Aboriginal and Torres Strait Islander communities, a PAR model has been shown to assist in overcoming potential barriers created through mistrust, gives appropriate recognition to Indigenous 
knowledge and perspectives, and fosters information collection that accords with Indigenous communication styles and cultural constraints on knowledge sharing (Williams \& Tomison, 2013). Applying these notions to yet another cultural situation, Letiecq and Bailey, (2004) noted the importance of building and reaffirming relationships when conducting research with Native American (American Indian) groups. In common with Indigenous Australians, Native Americans have experienced historical injustices and misuse of traditional knowledge. Conducting research through PAR approaches has been found to be an effective way of working through these barriers and fostering trust and mutual exchange of knowledge (Letiecq \& Bailey, 2004), as we have found with the JOY evaluation.

\section{Constraints to Practice}

As noted at the outset, this paper aimed to provide an operational example of attempts to apply a PAR process to the evaluation of a multi-faceted government project in a cross-cultural, cross-language context. The evaluators adopted PAR approaches within a realist evaluation framework and these approaches helped to progress the evaluation in an inclusive, collaborative way that assisted with overcoming some of the cultural and language differences between evaluators and program stakeholders and participants. At the same time, the process highlighted some of the limitations of trying to apply genuinely collaborative and interactive approaches across these differences and across distance.

In an ideal application of PAR, evaluators and participants would meet regularly to reflect on their experiences and plan next steps. There would then be a process of implementation and observation, followed by further reflection and planning. While the JOY evaluation is able to incorporate these cycles, there are some very real limitations on the capacity of evaluators and participants to meet and discuss. While this can be partly overcome through regular video-conferencing between the AIC evaluators and key DJOP staff, it will not be practical to include the larger group of participants in those discussions. Further, while these video-conferences can be conducted regularly, this will have to be held at fairly fixed intervals that may not align with times and stages that would be best suited to observation and reflection. The video-conferences may also be more structured and less conducive to free-ranging discussion than might be ideal for a PAR approach.

Differences in language will also tend to limit the scope of discussion and prevent sharing of some of the more subtly nuanced elements of reflection that might otherwise benefit the evaluation. Language differences will also tend to require the discussions to be structured and involve both participants and evaluators preparing some of the information material in advance of the discussions. This will also tend to constrain discussion and raises the potential for the evaluation process to be more fully guided and led by the evaluators than would be ideal. This carries an inherently related risk of the process being overly influenced by the evaluators' Western perspectives and modes of working. 
Some of the basic tenets of PAR require participants to keep and build records of reactions, judgements, impressions and to establish evidence of changes and improvements (McTaggart, 1989). The capacity of participants to do this, in a way that can be shared with evaluators, is limited in a cross-language context as records cannot be directly shared and there can be substantial costs involved in translating records. The need for translation may limit the scope to which records can be used to capture reactions and impressions. Apart from these issues, participants will be working in roles where they will face competing priorities and time pressures will tend to reduce the extent of their record-keeping. The problems of distance and different political and social environments within which the evaluators and participants are operating will reduce the ability of the evaluators to positively influence these aspects of the PAR process.

One further consideration that may arise in other cross-cultural evaluations, much more than it has for the JOY project, is the issue of power imbalances between evaluators and participants. This will typically arise when researchers and evaluators from mainstream organisations, particularly government agencies, work with Indigenous peoples. It is important to recognise and respect the impacts of past colonisation experiences and detrimental government policies as research practices that engendered distrust and current experiences of disadvantage is incumbent on evaluators. PAR approaches can help with empowering Indigenous peoples in the context of these impacts. Work on the JOY project raised the potential for evaluators to perpetuate a 'methodology of imperialism' (Said 1995, cited in Liamputtong 2010: 22) by imposing Western perspectives and paradigms on Eastern participants. However, the JOY participants are educated and qualified government staff members who are relatively empowered compared to participants in other cross-cultural contexts, such as members of Indigenous communities. As has been described above, the evaluators deliberately adopted approaches to reduce imbalances as much as possible within the constraints imposed by the contract and evaluation environment in order to set up a process of real partnership between the AIC and DJOP who each brought valuable knowledge and expertise to the evaluation process.

\section{Conclusions and Implications}

Participatory ways of working are particularly important in cross-cultural contexts as they afford deeper levels of knowledge to emerge and become incorporated into the evaluation process, knowledge that may not otherwise transfer readily across cultural and linguistic divides. External evaluators, while bringing a different perspective and expertise, cannot have the same nuanced understanding of an intervention or its targets as those engaged continually in developing and implementing an intervention and in resolving the myriad of issues that typically arise in conducting this work. By adopting a true partnership approach, including the knowledge, expertise and insights of intervention participants in the evaluation, a more richly informed and valid evaluation outcome can be produced. Importantly, the PAR approach supports respectful ways of working together and can bridge distrust and apprehension between evaluators and stakeholders. 
The approach taken in the JOY evaluation, and the initial workshop described here, would have utility in a wide range of cross-cultural situations. It highlights the value of capacity building and PAR approaches combined with the use of structured tools, such as program logic models, when conducting evaluations in cross-cultural contexts. PAR and program logic approaches allowed program stakeholders, who held rich and deep knowledge about the program and its target audience, to share their knowledge with the evaluators in a mutually beneficial and trusting way that placed explicit value on the knowledge shared by all participants. The cycles of reflection, information collection and action implemented through PAR allowed this knowledge to be gathered, checked and evaluation processes adjusted in a way that effectively disabled some of the conceptual barriers that can arise when working across different languages and cultures.

The structured and succinct nature of program logic models proved to be an effective tool for communication during the JOY workshop that could be similarly applied in other cross-cultural and cross-language situations. Program logic models help to bring together large amounts of information in an accessible format by capturing the key elements and activities required for a program to operate effectively. The presentation of expected outcomes allows stakeholders to readily engage with and to help define a project's outcomes, and that critical reflection can build greater understanding of the program and the true nature of its objectives among both stakeholders and evaluators.

Yet it is recognised that there are limitations to the application of PAR approaches within the practical constraints of this evaluation, and with other evaluations operating in cross-language contexts and over distance. Evaluators and participants can work together to overcome those limitations, but the extent to which difference and distance limit the ability to freely share information, observations, reactions and perceptions may depend on how best practice PAR approaches can be utilised. Despite this, PAR provides an appropriate model for making genuine attempts to ensure collaborative, inclusive and respectful evaluation practice.

While there is still considerable work to be done on the evaluation of the JOY project, the foundations put in place during the workshop in Bangkok and the continuing application of participatory ways of working together are expected to yield positive benefits throughout the life of the evaluation. Feedback from workshop participants indicated clearly that they found the process worthwhile and considered themselves as active participants involved in the development and operation of the evaluation. That is, a sense of ownership was achieved. While the evaluators recognise that Thai culture emphasises politeness and respect, particularly for foreign visitors invited to share their knowledge and expertise, this feedback was encouraging and has been reinforced in subsequent communications.

As the evaluation progresses, the authors will gain clearer indications of whether the approach described in this paper was effective. As data and information becomes available, it will yield insights into whether the goals of shared understanding and engagement are being realised. A key benefit of PAR is that it provides a model for collaborative engagement to find ways of improving the process. Also, it is expected 
that by continuing to work through cycles of planning, implementation, review and improvement with our Thai colleagues, there remains considerable scope for achieving effective outcomes in this cross-cultural evaluation. In the longer term, we believe the approaches adopted will increase the likelihood that the evaluation findings will adequately and reliably reflect the outcomes that have been achieved through the project.

\section{References}

Baum, F., MacDougall, C. \& Smith, D. (2006). Participatory Action Research. Journal of Epidemiology and Community Health, 60(10): 854-867.

Borzycki, M., \& Baldry, E. (2003). Promoting integration: The provision of prisoner post-release services. Trends \& issues in crime and criminal justice, No. 262. Canberra: Australian Institute of Criminology.

Brydon-Miller, M., Kral, M., Maguire, P., Noffke, S., \& Sabhlok, A. (2011). Jazz and the Banyan Tree: Roots and Riffs on Participatory Action Research. In N. Denzin \& Y. Lincoln (Eds.), The SAGE Handbook of Qualitative Research (4th ed., pp. 387400). California: Sage Publications.

Burns, D. (2006). Evaluation in Complex Governance Arenas: the Potential of Large System Action Research. In B. Williams \& I. Imam (Eds.), System Concepts in Evaluation: An Expert Anthology (pp. 181-196). California: Edge Press.

Clark, H. \& Anderson, A.A. (2004). Theories of Change and Logic Models: Telling Them Apart. Paper presented at the American Evaluation Association, Atlanta, Georgia, 3-6 November 2004. Retrieved from http://www.theoryofchange.org/ library/presentations

Crane, P., \& O’Regan, M. (2010). On PAR: Using Participatory Action Research to Improve Early Intervention. Canberra: Department of Families, Housing, Community Services and Indigenous Affairs, Australian Government. Retrieved from http://www.dss.gov.au/our-responsibilities/housing-support/publicationsarticles/homelessness-youth/on-par-using-participatory-action-research-toimprove-early-intervention?HTML=

Frechtling, J. (2007). Logic Modeling Methods in Program Evaluation. California: John Wiley and Sons.

Funnell, S., \& Rogers, P. (2011). Purposeful Program Theory. San Francisco: Jossey-Bass.

Hecker, R. (2008). Participatory action research as a strategy for empowering Aboriginal health workers. Australian and New Zealand Journal of Public Health, 21(7), 784-788.

Kemmis, S., \& McTaggart, R. (2000). Participatory Action Research. In N. Denzin \& Y. Lincoln (Eds.), Handbook of Qualitative Research (2nd Ed., pp. 567-605). California: Sage Publications. 
Letiecq, B.L., \& Bailey, S.J. (2004). Evaluating From the Outside: Conducting Crosscultural Evaluation Research on an American Indian Reservation. Evaluation Review, 28(4), 342-357.

Liamputtong, P. (2010). Performing Qualitative Cross-Cultural Research. Cambridge: Cambridge University Press.

McLaughlin, J.A., \& Jordan, G.B. (1999). Logic Models: A Tool for Telling Your Program's Performance Story. Evaluation and Program Planning, 22(1): 65-72.

McLaughlin, J.A., \& Jordan, G.B. (2004). Using Logic Models. In J.S. Wholey, H.P. Hatry, \& K.E. Newcome. (Eds.), Handbook of Practical Program Evaluation. (pp.7-33). San Francisco, CA: Jossey-Banks.

McTaggart, R. (1989). 16 Tenets of Participatory Action Research. Paper presented to the 3er Encuentro Mundial Investigacion Participatva [The Third World Encounter on Participatory Research], Managua, Nicaragua, 3-9 September 1989. Retrieved from http://www.caledonia.org.uk/par.htm

Pawson, R. \& Tilley, N. (1997). Realistic Evaluation. London: Sage Publications.

Stringer, E. (2014). Action Research (4th Ed.). California: Sage Publications.

Stufflebeam, D. (2004). Evaluation Design Checklist. Michigan USA: The Evaluation Center, Western Michigan University. Retrieved from http://www.wmich.edu/ evalctr/archive_checklists/evaldesign.pdf

Tomison, A.M. (2000). Evaluating Child Abuse Prevention Programs. National Child Protection Clearinghouse Issues in Child Protection no.12. Melbourne: Australian Institute of Family Studies. Retrieved from http://www.aifs.gov.au/ nch/pubs/issues/issues12/issues12.html

Tomison, A.M. (2004). Current Issues in Child Protection Policy and Practice: Informing the Northern Territory's child protection review. Darwin: NT Department of Health and Community Services. Retrieved from http:// digitallibrary.health.nt.gov.au/prodjspui/handle/10137/121

Whyte, W.F., Greenwood, D.J. \& Lazes P. (1991). Participatory Action Research: Through Practice to Science in Social Research. In W.F. Whyte (Ed.), Participatory Action Research. California: Sage Publications.

Williams, E. \& Tomison, A.M. (2013). Monitoring and Evaluating Community based Interventions for Children and Families in the Asia-Pacific Region. In R.N. Srivastava, R. Seth \& J. van Niekerk (Eds.), Child Abuse and Neglect: Challenges and Opportunities (pp.159-172). New Delhi: JayPee. 


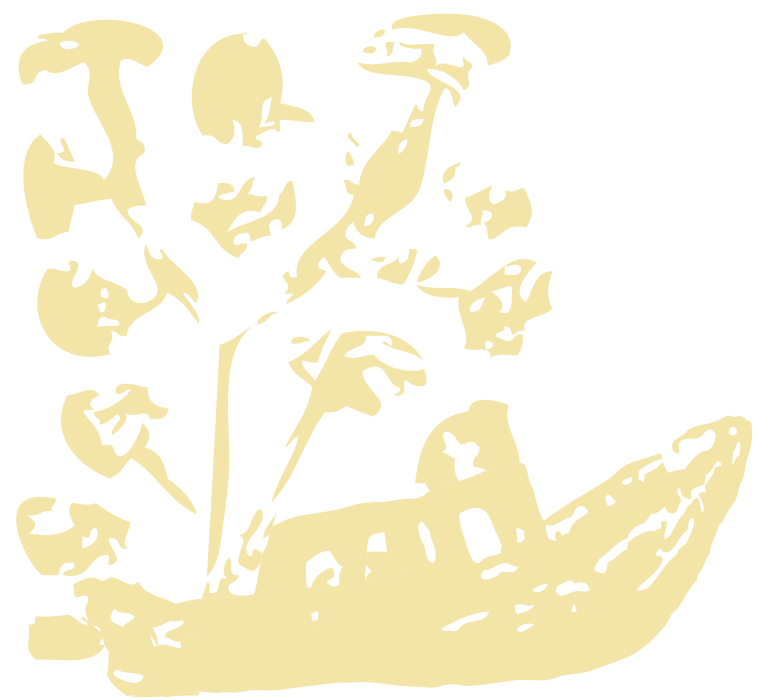

